\title{
Nanoscale Tuning of Grain Boundary Potential Barriers in Doped Strontium Titanate
}

\author{
Pradyumna L. Prabhumirashi ${ }^{1}$, Andrew R. Lupini ${ }^{2}$, Stephen J. Pennycook ${ }^{2}$ and Vinayak P. Dravid ${ }^{1}$ \\ ${ }^{1}$ Department of Materials Science \& Engineering, Northwestern University, Evanston, IL 60208. \\ ${ }^{2}$ Solid State Division, Oak Ridge National Laboratory, Oak Ridge, TN 37831.
}

Perovskite oxides are systems exhibiting a broad spectrum of technologically important properties. In particular, there has been a substantial interest in exploiting their functionality for superconductivity, ferromagnetism, magneto-resistance, ionic conductivity, and as next generation dielectrics [1]. Over the years it has become very clear that nanoscale features of interfaces in such materials often influence bulk phenomena and interface mediated properties. The electrical activity of such interfaces originates from a convoluted yet tractable interplay among numerous interfacial variables, including local atomic structure, defect chemistry and resultant localized changes in electronic structure. This is the basis for formation of interfacial trapped charge, and space-charge potential, which modify substantially under applied bias. An excellent example of this observed in electroceramics, where, the trapping of charge at interfaces has a decisive influence on the electrical transport properties through the formation of electrostatic potential barriers [2].

Our study utilizes oriented bicrystals of $\mathrm{SrTiO}_{3}$ as model systems to understand the nonlinear transport, capacitance and space-charge phenomena [3]. Direct current electrical measurements of individual grain boundaries in $\mathrm{Nb}$ doped $\mathrm{SrTiO}_{3}$ bicrystals reveal a highly resistive and non-linear behavior compared to single crystals due to the presence of internal potential barrier (FIG. 1). It is possible to 'tune' this barrier by selectively doping the grain boundary with transition metals, such as manganese and vanadium. Presently we are studying the effect of aliovalant solutes, such as Mn, on grain boundary barrier characteristics. Mn acts as ' $\mathrm{B}$ ' site substitutional solute in the $\mathrm{ABO}_{3}$-type (perovskite) structure replacing $\mathrm{Ti}^{4+}$. By tuning processing parameters one can manipulate variable valence states exhibited by $\mathrm{Mn}\left(\right.$ as $\mathrm{Mn}^{2+}$ and $\mathrm{Mn}^{3+}$ ) in order to tailor grain boundary barrier characteristics, particularly $\mathrm{F}_{\mathrm{B}}$ (barrier height).

In this paper, we use a combination of doping and heat treatment procedures to tune the grain boundary barriers in $\mathrm{SrTiO}_{3}$. The tuning occurs as a result of change in the defect chemistry specifically at interfaces. Oxygen vacancy distribution plays a vital role in the defect reactions. It has been shown that oxygen vacancies are energetically favorable at interfaces in $\mathrm{SrTiO}_{3}$ [4]. Preliminary experiments, done on Ni bulk doped $\mathrm{SrTiO}_{3}$ polycrystals; show that it is possible to fill in these oxygen vacancies using strong oxidizing conditions (FIG. 2 and FIG. 3). Implications of this in terms of resulting electronic structure at the interface and transport properties are being explored. Experiments are being done on doped bicrystalline $\mathrm{SrTiO}_{3}$. The use of bicrystals would allow us to accurately quantify the response (for example EELS) from a single GB that has a well defined structure. The presentation will highlight nanoscale characterization of such interfaces tuned with dopants and heat treatments. Electron holography is being used as a bulk sensitive tool to directly image and quantify the magnitude, sign, and spatial extent of electrical potential and charge across the interface [5,6]. Electrical measurements will be used in conjunction with high-spatial-resolution EELS, atomicresolution Z-contrast imaging and electron holography to obtain predictive structure-property correlations in $\mathrm{SrTiO}_{3}$. 
1. R. Waser and R. Hagenbeck, Acta Materialia 48, 797-825 (2000).

2. K. D. Johnson and V. P. Dravid, Interface Science 8, 189-198 (2000).

3. V. Ravikumar, R. P. Rodrigues, and V. P. Dravid, Physical Review Letters 75, 4063-66 (1995).

4. M. Kim, G. Duscher, N. D. Browning, K. Sohlberg, S. T. Pantelides, and S. J. Pennycook, Physical Review Letters 86, 4056-4059 (2001).

5. V. Ravikumar, R. P. Rodrigues, and V. P. Dravid, Journal of the American Ceramic Society 80, 1117-30 (1997).

6. K. Johnson and V. Dravid, Applied Physics Letters 74, 621-623 (1999).

7. This research is supported by the U. S. Department of Energy, Office of Basic Energy Sciences, under Grant No. DE FG02-92ER45475.

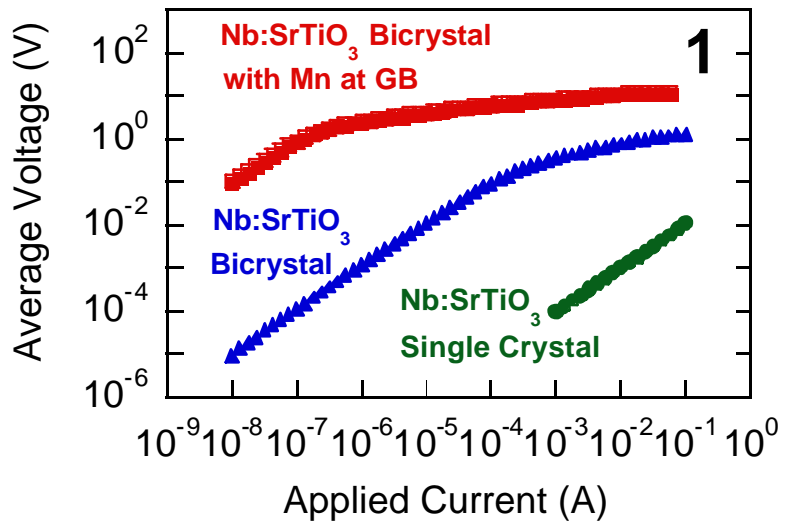

Fig. 1 - Comparison of the 4 probe I-V measurements of single crystalline versus bicrystalline (pristine and GB enriched) $\mathrm{Nb}$ STO. Pristine bicrystal has resistivity about $10^{3}$ times higher while Mn GB enriched bicrystal has about $10^{6}$ times higher resistivity than single crystal $\mathrm{SrTiO}_{3}$.

\section{Ni-doped STO}

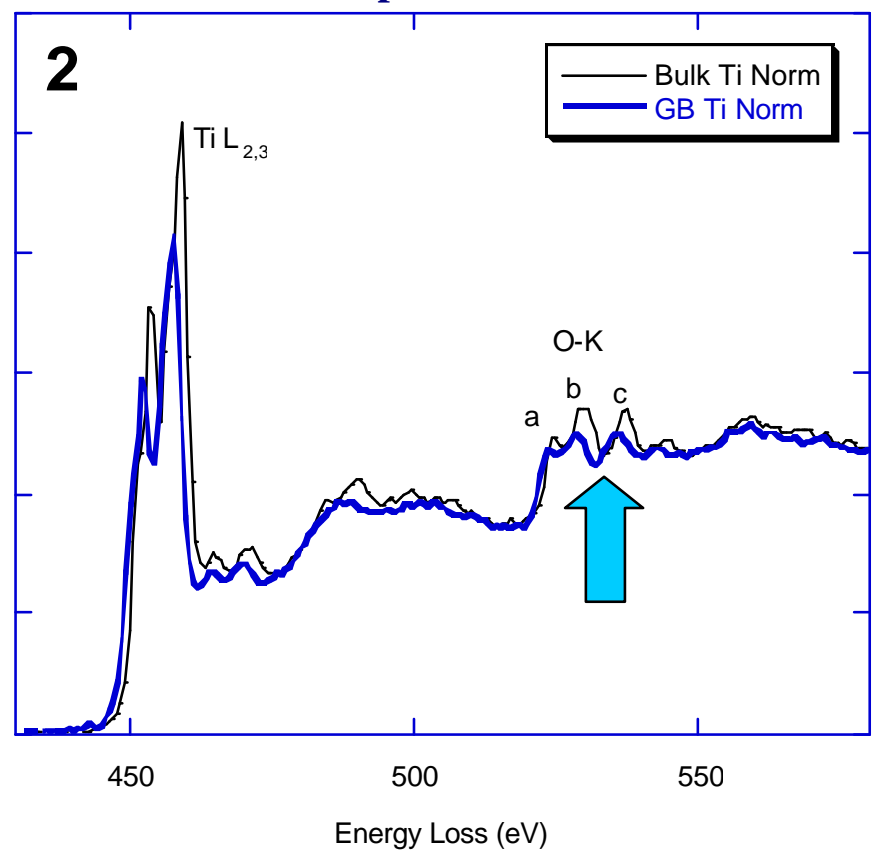

Ni-doped STO after Oxidation

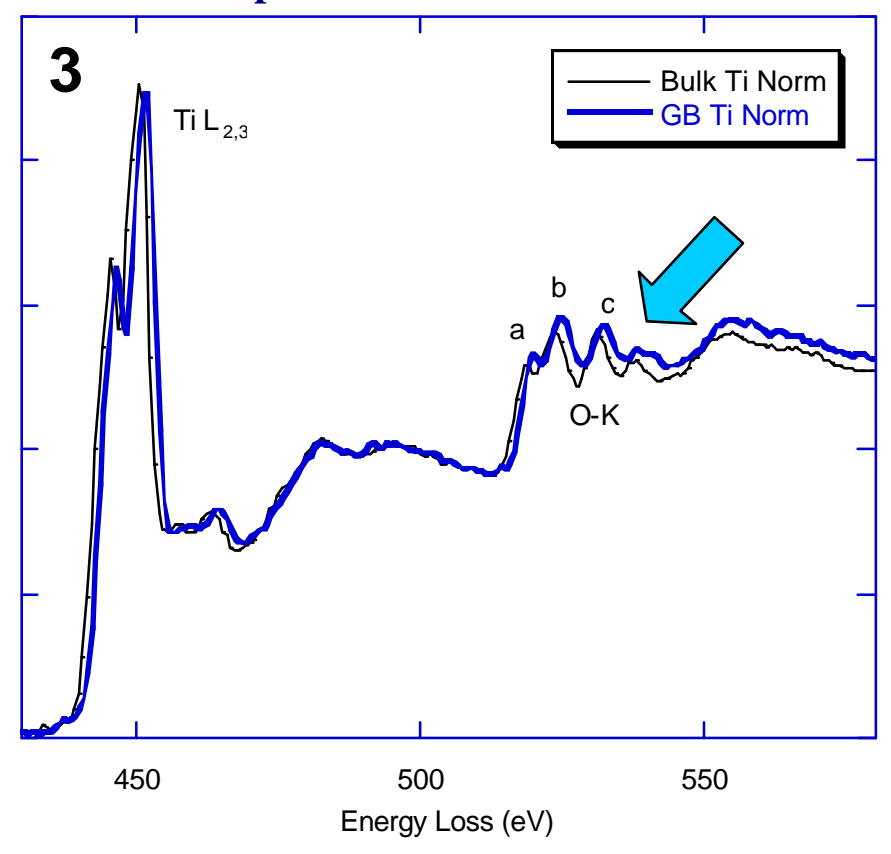

Fig. 2 and 3 - Comparison of the EEL spectra on and off the grain boundary in Ni doped $\mathrm{SrTiO}_{3}$. Figure 3 compares similar spectra in oxidized Ni doped $\mathrm{SrTiO}_{3}$. Ni-bulk doped $\mathrm{SrTiO}_{3}$ polycrystalline samples were oxidized at $1100{ }^{\circ} \mathrm{C}$ in air. Notice the differences in the $\boldsymbol{O}$-K edge fine structure as well as $T i \boldsymbol{L}_{2} / \mathbf{L}_{3}$ ratio. In the pristine sample, the GB O-K edge (marked by points $\mathrm{a}, \mathrm{b}$, and $\mathrm{c}$ ) has a lower intensity than its bulk counterpart. This changes significantly when the sample is treated in oxygen atmosphere. It is also observed that when oxidized, the $\mathrm{Ti}_{2} / \mathrm{L}_{3}$ ratio at the GB becomes almost identical to that in the bulk. Both spectra are normalized using Ti $\mathrm{L}_{2,3}$ edge continuum (about $30 \mathrm{eV}$ before the onset of the O-K edge). 